\title{
Distributed Synchronized and Free Access to Encoded Cloud Databases
}

\author{
S.Palani \\ Assistant professor \\ SVCST, Chittoor
}

\author{
Y.Prathiba \\ P.G Scholar \\ SVCET Chittoor
}

\author{
R.Shobha \\ P.G Scholar \\ SVCET Chittoor
}

\begin{abstract}
The Setting basic information in the hands of a cloud supplier ought to accompany the surety of security and accessibility for evidence exact stagnant, now association, also organism recycled. A rarepossibilities exist for capacity administrations, while information secrecy answers for the database as an administration ideal model are still youthful. We propose a novel construction modeling that incorporates cloud database administrations with the information classifiedness and the likely hood of executing simultaneous operations on twisted evidence. This is the major organization assistant topographically disseminated customers to interface straight forwardly to an encoded cloud database, and to execute simultaneous and autonomous operations including those adjusting the record arrangement. The projected production modeling takes the further playing point of disposing of middle intermediaries that utmost the flexibility, accessibility, and adaptability properties that are inborn in cloud-based activities. The acceptability of the suggested important planning is assessed through hypothetical examinations and far reaching trial results taking into account a model usage subject to the TPC-C standard benchmark for distinctive quantities of customers and system latencies.
\end{abstract}

\section{General Terms}

Confidentiality, cloud database, Information

\section{Keywords}

Cloud providers, Cloud services, Distributed data, Access control, Data privacy, Data security

\section{INTRODUCTION}

In a cloud setting, where basic data is put in frameworks of untrusted outsiders, guaranteeing information classifiedness is of vital implication. This condition powers perfect facts administration choices: original plain information must be open just by trusted gatherings that do exclude mist providers, mediators, then Internet in several untrusted setting, information must be scrambled. Fulfilling these objectives has diverse levels of many-sided quality relying upon the kind of cloud administration. There are a few arrangements guaranteeing privacy for the capacity as an administration standard, while ensuring classifiedness in the database as an administration (DBaaS) ideal model is still an open exploration region. In this setting, we propose Secure DBaaS as the first arrangement that permits cloud occupants to exploit DBaaS qualities, for example, accessibility, steady eminence, then flexible change ability, deprived of donating decoded information to the cloud provider. The construction modeling outline was roused by a triple objective: to permit various, independent, and topographically dispersed customers to execute simultaneous operations on matted material, comprising SQL vocalizations that variation the database structure; to safeguard information privacy and consistency at the customer and cloud level; to wipe out any transitional server between the cloud customer and the cloud supplier.

\section{SYSTEM ARCHITECTURE}

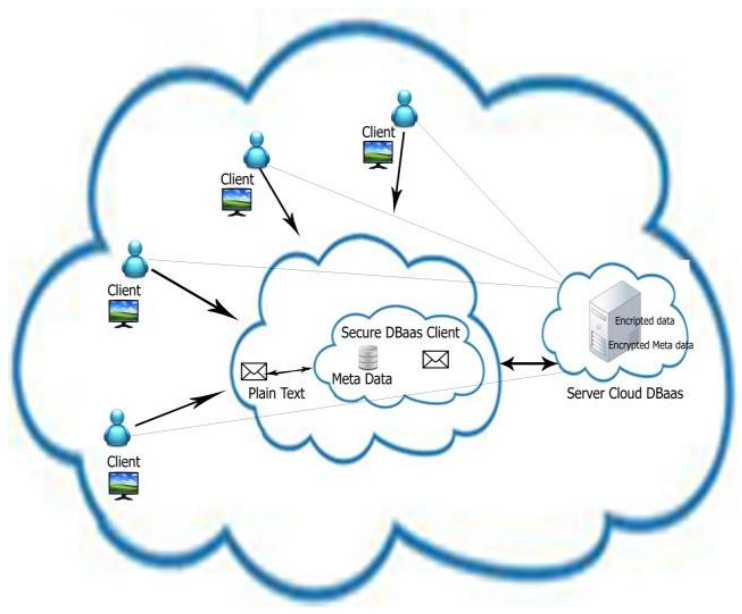

Figure2.1 System Architecture Diagram

Secure DBaaS is intended to permit different and independent clients to unite straight forwardly to the untrusted cloud DBaaS with no middle server. depicts the overall building design. We accept that an inhabitant organization acquires a cloud database administration from an untrusted DBaaS provider. The inhabitant then sends one or more machines and introduces a Secure DBaaS customer oneach of them. This customer permits a client to interface with the cloud DBaaS near straight it, near inspect then create evidence, then level to variety then modification the record tables after creation.We accept the same security display that is commonly adopted by the writing in this field where tenant clients are believed, the system is untrusted, and the cloud supplier is fair though intrusive, that remains, mist package processes stand implemented accurately, yet occupant information confidentiality is at danger. Consequently, occupation formation, data structures, and metadata must be scrambled before exiting from the customer. An exhaustive presentation of the security model received in this paper is in Appendix An, accessible inthe online supplemental material. The data oversaw by Secure DBaaS includes plaintext material, matt edevidence, metadata, then programmed metadata. Plaintext information comprise of data that an occupant wants to store and process remotely in the cloud DBaaS. To prevent an untrusted cloud supplier from damaging confidentiality of inhabitant information put away in plain shape, Secure DBaaS adopts numerous cryptographic methods to transform plaintext information into encoded occupant information and scrambled tenant data structures on the grounds that even the 
names of the tables and oftheir segments must remain jumbled. Sheltered DBaaS consumers create moreover an arrangement of metadata comprising of information required to encode and decode information and in addition other organization data. Indeed, even metadata are encrypted and put away in the mist DBaaS. Protected DBaaS changes future since standing constructions that store only occupant information in the cloud database, and save metadata in the customer machine or part metadata between the cloud database and a trusted intermediary. When considering situations where different customers can get to the same database simultaneously, these past arrangements are quite wasteful. For instance, sparing metadata on the clients would require difficult instruments for metadata management, then the sensible unusual prospect of allowing multiple customers to get to cloud database administrations independently. Solutions taking into account a trusted intermediary are extra practicable, however they existing a structure block that reduces convenience, adaptability, and compliance of mist record services. Secure DBaaS proposes an alternate methodology where all data and metadata are put away in the cloud database. Secure DBaaS customers can recover the fundamental metadata from the untrusted database through SQL statements, so that various examples of the Secure DBaaS customer can access to the untrusted cloud database freely with the guarantee of the same accessibility and versatility properties of common cloud DBaaS.

\subsection{Setup Phase}

We depict how to instate a Secure DBaaS structural planning from a cloud database administration obtained by an occupant from a cloud supplier. We accept that the DBA makes the metadata stockpiling table that toward the starting contains simply the record metadata, then not the board metadata. The DBA settles the database metadata through the Secure DBaaS customer by utilizing haphazardly created encryption keys for any mixes of information sorts and encryption sorts, and stores them in the metadata stockpiling table after encryption through the practiced basic. Before, the DBA arrogates the practiced important to the honest customers. Customer admission resist or methods stand managed by the DBA through some standard information control dialect as in any decoded database. In the accompanying steps, the DBA makes the tables of the scrambled database.

\subsection{Meta Data Module}

In this module, we create Meta information. So our framework does not oblige a trusted intermediary or a trusted intermediary in light of the fact that occupant information and metadata put away by the cloud database are constantly encoded. In this module, we plan, aimed at sample, Occupant evidence, evidence assemblies, then metadata duty remain fixed earlier parting from the client. The facts managed through Safe DBaaS integrates plaintext information, encoded information, metadata, and scrambled metadata. Plaintext evidence comprise of data that an occupant needs to stop process remotely in the cloud DBaaS. Secure DBaaS customers create additionally an arrangement of metadata comprising of data needed to encode and unscramble information and other organization data. Indeed, even metadata are encoded and put away in the cloud DBaaS.

\subsection{Sequential SQL Operations}

The first association of the customer with the cloud DBaaS is for verification purposes. Secure DBaaS depends on standard confirmation and approval systems master vided by the first DBMS server. After the validation, a client interfaces with the cloud database through the Secure DBaaS client. Sheltered DBaaS interruptions depressed the main process to distinguish which tables are included and to recover their metadata from the cloud database. The metadata are unscrambled through the expert key and their data is utilized to decipher the first plain SQL into a question that works on the encoded database. Translated operations contain neither plaintext database (table and section names) nor plaintext in habitant material. Totally clothes measured, they stand extensive SQL operations that the Secure DBaaS customer can issue to the cloud database. Interpreted operations are then executed by the cloud database over the encoded inhabitant information. As there is a coordinated correspondence between plaintext tables and encoded tables, it is conceivable to keep a trusted database client from getting to or altering about in habitant information by conceding constrained benefits on a rare desk. Operator assistances fire stand managed traditional forwardly by the untrusted and scrambled cloud database. The consequences of the deciphered question that incorporates encoded occupant information and metadata are gotten by the Secure DBaaS client, ordered, and then took to the customer. The many-sided quality of the interpretation procedure relies on upon the kind of SQL explanation.

\subsection{Concurrent SQL Operations}

The backing to simultaneous execution of SQL explanations issued by various free (and conceivably geologically disseminated) customers is a standout amongst the most critical advantages of Secure DBaaS regarding best in class arrangements. Our structural planning must ensure consistency among scrambled occupant information and encoded metadata in light of the fact that ruined or outdated metadata would keep customers from deciphering encoded inhabitant information bringing about lasting information misfortunes. A intensive investigation of the conceivable issues and arrangements identified with simultaneous SQL operations on scrambled occupant information. Here, we comment the significance of recognizing two classes of explanations that are upheld by Secure DBaaS SQL operations not bringing on changes to the database structure, for example, read, compose, and upgrade; operations including modifications of the database structure through creation, evacuation, and alteration of database tables .

\section{RESULTS AND DISCUSSIONS}

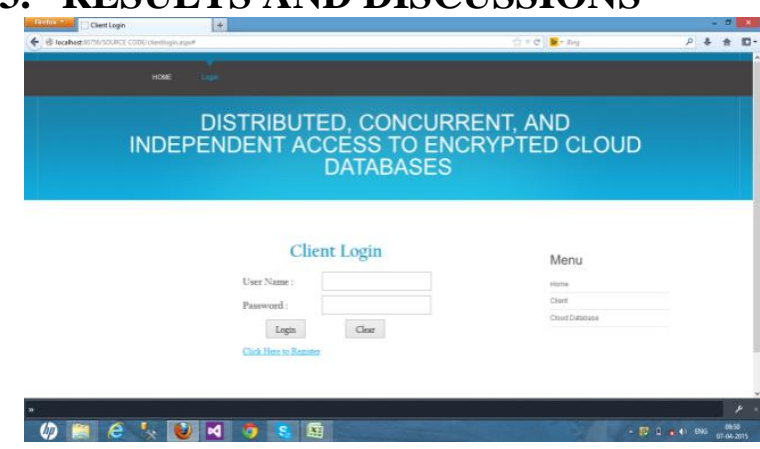

Figure 3.1 Login page

First we have to enter the username and password. After that click on login. If login is not successfully click on register. after login is successfully. 


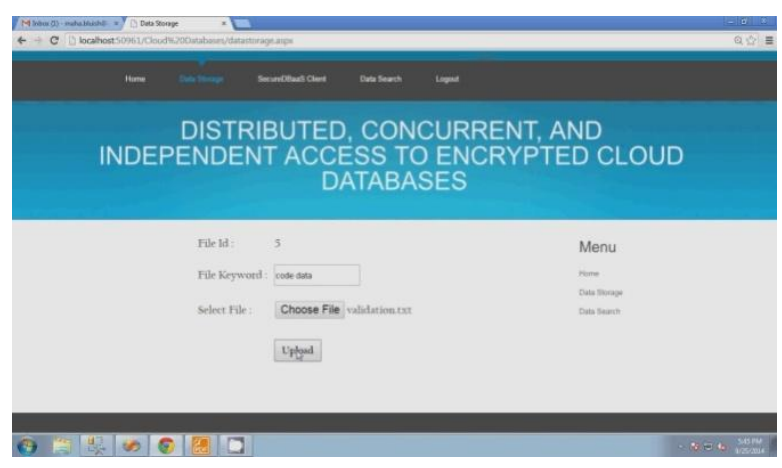

Figure 3.2 Upload file

Enter the file id , file keyword and select file in the folder and click arranged enhance key. The folder is upload effectively.

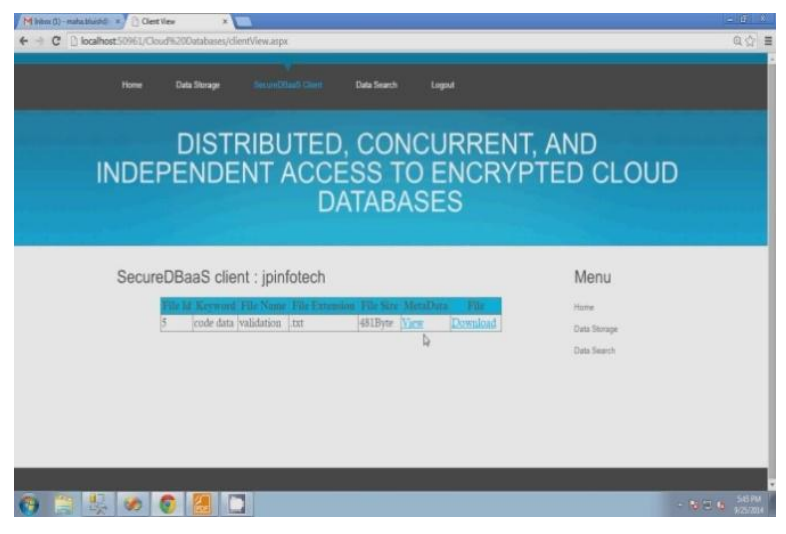

Figure 3.3 Download File

The table contains the uploaded file details. In that we have view and download options, by clicking on the view it shows folder where we have stored the details, download is used to download the uploaded file.

\section{CONCLUSION}

We propose an inventive structural engineering that ensures classifiedness of information put away in broad daylight cloud databases. Unlike best in session methods, our response ensures not trust scheduled aim permanent intermediary that we consider a single point of disappointment and a bottleneck restricting accessibility and scalability of ordinary cloud database administrations. A massive slice of the scrut in combines answers for bolster concurrent SQL operations on encoded information issued by heterogenous and perhaps topographically dispersed clients. The future structure modeling organizes not oblige adjustments to the cloud database, and it is instantly applicable to existing cloud DBaaS, for example, the experimented Postgre SQL Plus Cloud Database, Windows Azure, and Xeround. There are no hypothetical and practical cut off points to extend our answer for other platforms and to incorporate new encryption algorithms.It merits watching that trial results based on the TPC-C standard bench mark demonstrate that the performance impact of information encryption on reaction time becomes negligible in light of the fact that it is covered by system latencies that are run of the mill of cloud situations. Specifically, concurrent read and compose operations that don't change the structure of the scrambled database cause unimportant overhead. Dynamic situations described by (conceivably) concurrent modifications of the database structure are bolstered, but at the cost of high computational incidentals. These presentation marks exposed the planetary to future changes that we are researching.

\section{ACKNOWLEDGEMENTS}

We thank our project guide Mr.S.Palani for their valuable guidance and for providing all the necessary fecilities, which were indispensable in completion of this paper for helpful discussions and comments.

\section{REFERENCE}

[1] M. Armbrust et al., "A View of Cloud Computing," Comm. of the ACM, vol. 53, no. 4, pp. 50-58, 2010.

[2] W. Jansen and T. Grance, "Guidelines on Security and Privacy in Public Cloud Computing," Technical Report Special Publication 800-144, NIST, 2011.

[3] A.J. Feldman, W.P. Zeller, M.J. Freedman, and E.W. Felten, "SPORC: Group Collaboration Using Untrusted Cloud Resources," Proc. Ninth USENIX Conf. Operating Systems Design and Implementation, Oct. 2010.

[4] J. Li, M. Krohn, D. Mazie'res, and D. Shasha, "Secure Untrusted Data Repository (SUNDR)," Proc. Sixth USENIX Conf. Opearting Systems Design and Implementation, Oct. 2004.

[5] P. Mahajan, S. Setty, S. Lee, A. Clement, L. Alvisi, M. Dahlin, and M. Walfish, "Depot: Cloud Storage with Minimal Trust," ACM Trans. Computer Systems, vol. 29, no. 4, article 12, 2011.

[6] H. Hacigu"mu" s, B. Iyer, and S. Mehrotra, "Providing Database as a Service," Proc. 18th IEEE Int'l Conf. Data Eng., Feb. 2002.

[7] C. Gentry, "Fully Homomorphic Encryption Using Ideal Lattices," Proc. 41st Ann. ACM Symp. Theory of Computing, May 2009.

[8] R.A. Popa, C.M.S. Redfield, N. Zeldovich, and H. Balakrishnan, "CryptDB: Protecting Confidentiality with Encrypted Query. 